\title{
Probing Electronic Dynamics during Photochemical Reactions
}

\author{
Andres Tehlar, Peter M. Kraus, and Hans Jakob Wörner ${ }^{\S \star}$ \\ §Grammaticakis-Neumann Prizewinner 2012
}

\begin{abstract}
This review discusses a new method for probing the evolution of the valence-electron structure of molecules during chemical reactions. The method relies on the interaction of an intense infrared laser pulse with molecules that results in the emission of attosecond pulses $\left(1\right.$ as $\left.=10^{-18} \mathrm{~s}\right)$ in a process known as high-harmonic generation. Time-resolved high-harmonic spectroscopy measures the phase and amplitude of attosecond pulses emitted from the reacting molecules through interference with the emission from the unexcited molecules. This coherent detection mechanism provides a high sensitivity to small excitation fractions and direct access to both the amplitude and the phase of attosecond pulses, the latter of which is otherwise very difficult to measure. These observables reveal several complementary aspects of excited-state photochemical dynamics such as dissociation, adiabatic wave-packet evolution and conical intersection dynamics.
\end{abstract}

Keywords: Attosecond spectroscopy · Conical intersections · High-harmonic generation · Photodissociation · Transient grating

\section{Introduction}

The outcome of all chemical reactions is controlled by the valence electronic structure of the reacting molecules. Understanding the selectivity of chemical processes and the reactivity of molecules would therefore greatly benefit from a direct experimental access to electronic structure. Since the elementary steps of chemical reactions take place on femtosecond to attosecond ( $1 \mathrm{fs}=1000$ as $=10^{-15} \mathrm{~s}$ ) time scales, any experimental method aiming at resolving these fundamental events must offer an adequate temporal resolution. Since the relevant length scales are those of a chemical bond, a spatial resolution on the order of 1 Angström is simultaneously required. Both conditions can be met by adequate applications of the rapidly developing tools of attosecond science.

The basic mechanism of attosecond pulse generation can be qualitatively understood as a sequence of three steps: ionization of a molecule by the electric field of an intense femtosecond laser pulse $\left(\sim 10^{14} \mathrm{~W} / \mathrm{cm}^{2}\right)$, acceleration of the liberated electron by the laser field, and finally recombination of electron and parent ion

\footnotetext{
${ }^{*}$ Correspondence: Prof. Dr. H. J. Wörner ETH Zürich

Laboratory for Physical Chemistry

Wolfgang-Pauli-Str. 10

$\mathrm{CH}-8093$ Zürich

Tel.: +41446334412

E-mail: woerner@phys.chem.ethz.ch
}

accompanied by emission of an attosecond pulse. ${ }^{[1,2]}$ The achieved spatial resolution is determined by the de-Broglie wavelength of the recombining photoelectron, which is on the order of $1-2 \AA$ in typical experiments. ${ }^{[3-5]}$ A temporal resolution of a few femtoseconds can be reached by launching a dynamical process with a first, weak, laser pulse followed by the generation of attosecond pulses in the form of high-order harmonics from a second, precisely delayed, intense laser pulse. ${ }^{[4,6-8]}$ In special cases, where the dynamics of interest can be prepared by strong-field ionization, a sub-femtosecond resolution can be achieved using a single high-harmonicgeneration pulse by exploiting the unique relationship between electron excursion time and emitted photon energy. ${ }^{[9-12]}$

The application of high-harmonic spectroscopy to studying photochemical dynamics, termed time-resolved high-harmonic spectroscopy (TRHHS), has been described in refs. [13-18] and will be reviewed here. So far, TRHHS has been applied to studying the photodissociation of $\mathrm{Br}_{2},[13,14,17]$ the conical intersection dynamics and subsequent dissociation of $\mathrm{NO}^{,[15-17]}$ both following photoexcitation at $400 \mathrm{~nm}$, as well as the non-adiabatic photodissociation of $\mathrm{CH}_{3} \mathrm{I}$ and $\mathrm{CF}_{3} \mathrm{I}$ following photoexcitation at 267 nm. ${ }^{[18]}$ Theoretical models of increasing detail have also been developed and are described in refs. [14,16,17]. The experimental results, through comparisons with the theoretical models, have demonstrated the main principles and sensitivities of TRHHS.

\section{Experiment}

Experiments using time-resolved highharmonic spectroscopy have been performed either in a collinear pump-probe geometry ${ }^{[14]}$ or using the transient-grating configuration. ${ }^{[13,15,16,18]}$ While the interference between high-harmonic emissions of excited and unexcited molecules is exploited in both cases, the transient-grating configuration, illustrated in Fig. 1, provides the intensity of the diffracted radiation as an additional observable that complements the intensity of the undiffracted radiation. While the latter has been shown to be formally identical in collinear and transientgrating pump-probe experiments carried out with identical spatially-averaged excitation fractions, ${ }^{[17]}$ the diffracted radiation has an enhanced contrast. Whereas the undiffracted radiation arises from signals of excited and unexcited molecules weighted by their relative populations, the diffracted radiation results from an interference of emissions with equal weights and thus enhanced contrast. An additional advantage of the transient-grating configuration is that both phase and amplitude of the highharmonic emission of excited molecules can be obtained. ${ }^{[13,19]}$ This is achieved by using the time-independent emission of the unexcited molecules as a reference.

A schematic drawing of the transientgrating setup is shown in Fig. 1. Two temporally synchronized excitation pulses cross at a small angle in a supersonic molecular beam where they build up an interference grating. The molecules located inside the bright fringes of the transient 


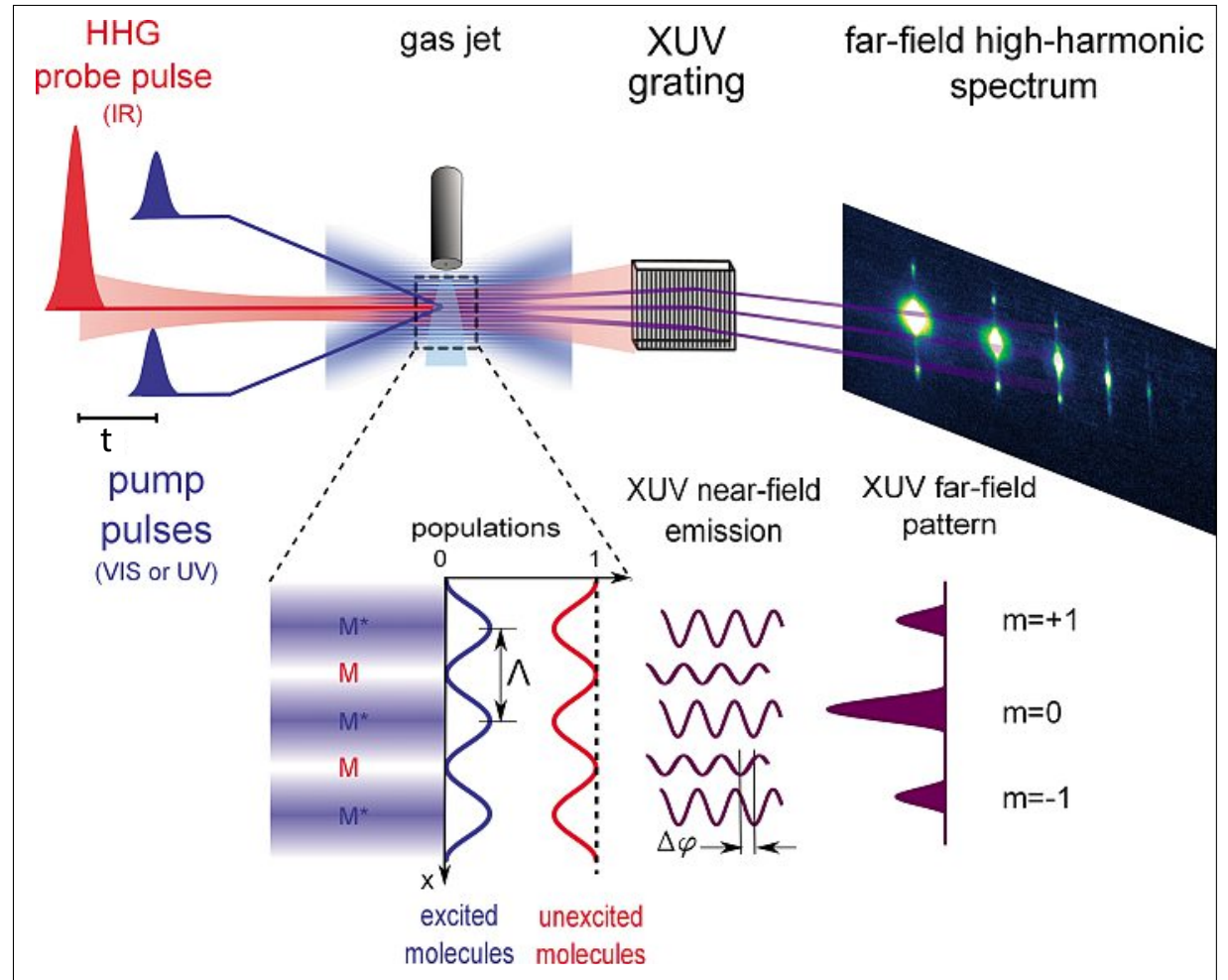

Fig. 1. Experimental setup of time-resolved high-harmonic spectroscopy in a transient-grating configuration. See main text for the detailed description.
Different wavelengths are separated using an XUV grating, placed between the source (transient grating) and the detector, which diffracts the light in a direction perpendicular to $x$. Since the emitted frequencies correspond to the odd harmonics of the generating field, the spectrally resolved far-field image on the detector consists of undiffracted spots $(\delta(x /(\lambda L))$ term in Eqn. (3), the zero-order diffraction of the transient grating $m=0$ ) and vertically displaced diffracted spots $(\delta(x /(\lambda L) \pm 1 / \Lambda)$ terms in Eqn. (3), the first order diffraction $m= \pm 1$ ) for each harmonic. Around time zero, when the pump and probe pulses interact simultaneously with the sample, additional spots arise, which can be explained by the absorption of $n_{1}$ photons from the probe pulse and $n_{2}$ photons from the pump pulses, leading to emission in directions between the two interacting beams. ${ }^{[20]}$ This phenomenon can be described as wavemixing and the observed emission spots can be labeled according to the number of absorbed pump-pulse photons as $w m=$ $\pm 1, \pm 2, \ldots$. Using perpendicularly polarized pump and probe pulses minimizes wavemixing. ${ }^{[20]}$ Fig. 2 shows a typical transient- grating are excited whereas no excitation occurs in the dark fringes. Since the probability of one-photon absorption scales linearly with the intensity, the excitation fraction $r$ is a sinusoidal function of the spatial coordinate $x$ of the grating with a periodicity of $\Lambda$ :

$$
r_{\mathrm{e}}=R \cdot \frac{1}{2}\left(\cos \left(\frac{2 \pi x}{\Lambda}\right)+1\right)
$$

where $R$ is the excitation fraction in the bright fringes. Some time delay $t$ after the excitation pulses, an intense $\left(I \sim 10^{14} \mathrm{~W} /\right.$ $\mathrm{cm}^{2}$ ) infrared probe pulse generates high harmonics in the structured sample, leading to a spatially modulated emission for each generated harmonic $q$ (atomic units are used unless otherwise specified):

$$
E_{\text {near field }}=d_{\mathrm{g}}\left(1-r_{\mathrm{e}}\right)+d_{\mathrm{e}} r_{\mathrm{e}}
$$

where $d_{\mathrm{g}}$ are the complex spectral components of high-harmonic emission from the ground state (subscript g) or excited state (e), respectively. For simplicity, we assume here that the pump and probe pulses are significantly shorter than the investigated dynamics. As a result of diffraction, the emitted electric field in the optical far-field is the spatial Fourier-transform of the nearfield emission:

$$
\begin{aligned}
E_{\text {far field }}= & \left(d_{\mathrm{g}}+\frac{R}{2}\left(d_{\mathrm{e}}-d_{\mathrm{g}}\right)\right) \cdot \delta\left(\frac{x}{\lambda L}\right) \\
& +\frac{R}{4}\left(d_{\mathrm{e}}-d_{\mathrm{g}}\right) \cdot \delta\left(\frac{x}{\lambda L}-\frac{1}{\Lambda}\right) \\
& +\frac{R}{4}\left(d_{\mathrm{e}}-d_{\mathrm{g}}\right) \cdot \delta\left(\frac{x}{\lambda L}+\frac{1}{\Lambda}\right),
\end{aligned}
$$

grating measurement using $267 \mathrm{~nm}$ pump and $800 \mathrm{~nm}$ probe pulses ${ }^{[18]}$ at $t<0$, where the sample is not excited (panel a), display- where $\delta$ is the Dirac delta function, $L$ is the distance from the source to the detector and $\lambda$ the wavelength of the radiation.

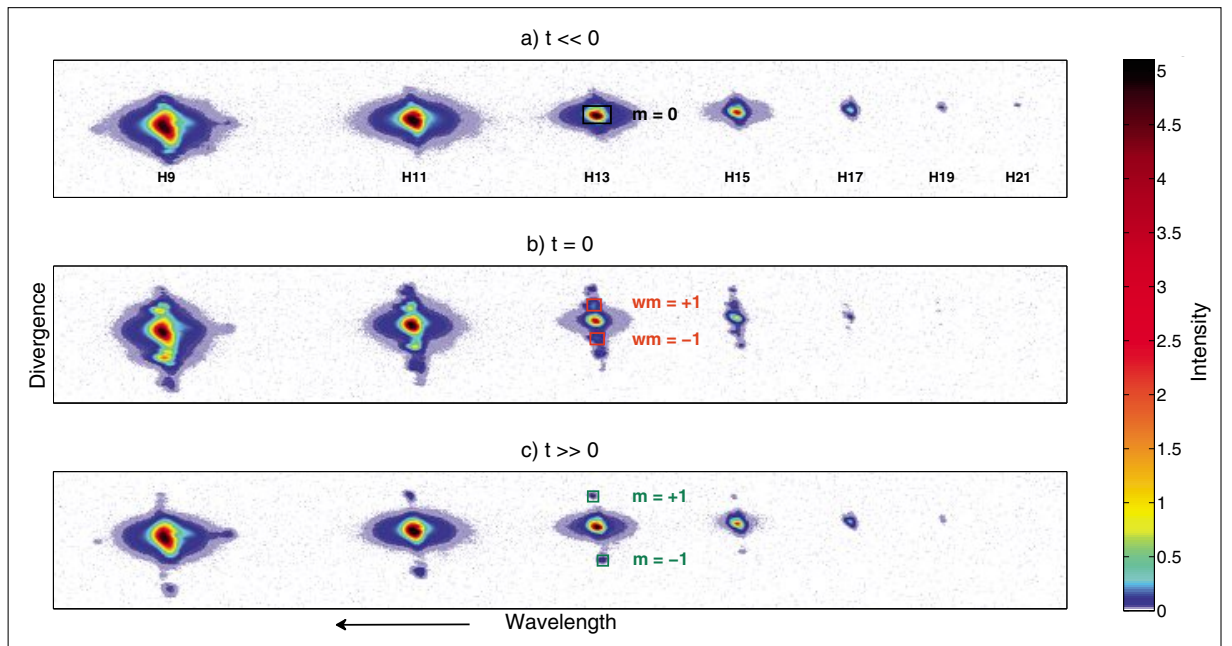

Fig. 2. Intensity distribution observed on the detector for different delay times $t$ between two synchronized excitation pulses with $\lambda_{\text {pump }}=267 \mathrm{~nm}$ and the high-harmonic-generating probe pulse centered at $\lambda_{\text {probe }}=800 \mathrm{~nm}$. In the upper panel (a), high harmonics are generated before the excitation grating is built up in the sample, leading to the absence of off-axis emission. In (b), recorded at temporal overlap of pump and probe pulses $(t=0)$ additional off-axis emission is visible that arises from wave mixing between the pump and probe pulses. In the lower panel (c), high-harmonic generation occurs in the presence of a grating of excited molecules resulting in first-order diffraction. 
ing no off-axis spots, $t=0$ (panel $\mathrm{b}$ ), where one observes both wavemixing and diffraction from the transient grating, and finally $t$ $>0$ (panel c), where only transient grating diffraction spots are visible.

While dynamics in the ground electronic state can be induced when intense resonant pump pulses are used (see e.g. ref. [13]), this situation can in general be avoided, in which case the intensities measured at the position of the undiffracted and diffracted spots are given by Eqns (4) and (5).

In the experiments, we normalize the signals in each harmonic order by dividing them through the undiffracted intensity at negative pump-probe delays $t$, i.e. $I_{m=0}(t<<$ $0)=\left|d_{\mathrm{g}}\right|^{2}$, define the relative amplitude $D$ and the phase difference $\Delta \varphi$ (Eqn. (6)) and thus show the normalized signals (Eqns (7) and (8)).

To interpret the variation of the phase and amplitude with pump-probe delay $t$, we have developed a model of highharmonic emission ${ }^{[17]}$ relying on the factorization of the complex amplitudes into three contributions. ${ }^{[1,2]}$ The complex spectral components of high-harmonic emission are expressed as the product of three steps, strong-field ionization of the molecule, propagation of the electron in the continuum and the recombination of the electron with its parent cation. Each of these steps contributes a phase and an amplitude to the emitted radiation, allowing us to approximate the transition dipole as Eqn. (9) where $\mathbf{R}$ collectively represents the internal nuclear degrees of freedom of the molecule, $i$ denotes the electronic state of the molecule which emits high harmonics, $f$ symbolizes the electronic state of the molecular cation during the high-harmonic generation (HHG) process, $\chi_{\mathrm{i}}$ is the nuclear wave function, $I^{\mathrm{if}}$ is the strong-field ionization rate from state $i$ to $f, I$ if is the vertical ionization potential from $i$ to $f, \tau$ the excursion time of the electron in the continuum during HHG, and $d^{\text {if }}$ the complex photorecombination matrix element. ${ }^{[17]}$ The term $\exp \left(-i I^{\text {if }}(\mathbf{R}) \tau\right)$ stems from the propagation of the electron in the continuum and plays an important role in the phase of high-harmonic emission. ${ }^{[21]}$ In adiabatic photodissociation processes involving a single electronically excited state, we use $d_{\mathrm{i}}=d_{\mathrm{e}}$. In cases involving multiple electronic states, as is often the case in polyatomic molecules such as $\mathrm{NO}_{2}$ discussed below, we use one $d$ for each diabatic state and sum the contributions as described in ref. [17].

\section{Results and Discussion}

We illustrate the principles of time-resolved high-harmonic spectroscopy by first discussing results on the photodissociation of $\mathrm{Br}_{2}$ molecules. Single-photon absorption at $400 \mathrm{~nm}$ excites the molecules from the $\mathrm{X}^{1} \sum_{\mathrm{g}}^{+}$ground state to the repulsive $\mathrm{C}$ ${ }^{1} \Pi_{1}$ excited state (Fig. 3a) thus creating a dissociative nuclear wave packet. Figs $3 b$ and $3 c$ show the experimental highharmonic intensities for the zeroth-and first-order diffracted signals, respectively. The zeroth-order signal first decreases

$$
I_{m=0}(t)=|E(t, x=0)|^{2}=\left|d_{\mathrm{g}}+\frac{R(t)}{2}\left(d_{\mathrm{e}}(t)-d_{\mathrm{g}}\right)\right|^{2}
$$

$$
I_{m= \pm 1}(t)=|E(t, x= \pm L \lambda / \Lambda)|^{2}=\frac{R^{2}(t)}{16}\left|d_{\mathrm{e}}(t)-d_{\mathrm{g}}\right|^{2}
$$

$$
d_{\mathrm{N}}=d_{\mathrm{e}} / d_{\mathrm{g}}=D \cdot \exp (i \Delta \varphi),
$$

$$
\begin{aligned}
I_{N, m=0}(t)=1 & +R(t) \cdot[D(t) \cos (\Delta \varphi(t))-1] \\
& +\frac{R^{2}(t)}{4} \cdot\left[1-2 D(t) \cos (\Delta \varphi(t))+D^{2}(t)\right]
\end{aligned}
$$

$$
I_{N, m= \pm 1}(t)=\frac{R^{2}(t)}{16} \cdot\left[1-2 D(t) \cos (\Delta \varphi(t))+D^{2}(t)\right]
$$

$$
d_{\mathrm{i}}(\lambda, t)=\int \mathrm{d} \mathbf{R}\left|\chi_{\mathrm{i}}(\mathbf{R}, t)\right|^{2} \sum_{\mathrm{f}} \sqrt{I^{\mathrm{if}}(\mathbf{R})} e^{-i I_{p}^{\mathrm{if}(\mathbf{R}) \tau}} d_{\mathrm{rec}}^{\mathrm{if}}(\mathbf{R})
$$

and then recovers to a harmonic-orderdependent level. The first-order diffraction signal displays a narrow local maximum at $t=0 \mathrm{fs}$ and then increases, reaching its maximum asymptotic value approximately $300 \mathrm{fs}$ after the excitation pulse. The intense maximum at $t=0 \mathrm{fs}$ is dominated by wavemixing because the pump and probe pulses were polarized parallel to each other in this experiment. A very simple theoretical model[13,14,17] has been found to capture the main observations of the experiment: the modulations of the harmonic emission in both the diffracted and undiffracted signals is dominated by the modulation of the contribution of the harmonic phase $e^{-i \Delta I_{p} \tau}$ resulting from the propagation of the electron in the continuum. ${ }^{[2]}$ Here, $\Delta I$ is the difference of the vertical ionization potentials of the excited state of the molecule and its ground state in its equilibrium geometry $\mathbf{R}_{\mathrm{e}}$ (as shown in the top panel of Fig. $3 \mathrm{a})$ and $\tau$ is the transit time of the continuum electron, which can be calculated for a given harmonic order using the classical model ${ }^{[1]}$ or the saddle-point approximation. ${ }^{[2]}$ Since the ionization potential is itself a function of the internuclear separation, so is $\Delta I$. These experiments are thus directly sensitive to the evolution of electronic binding energies as a function of the reaction coordinate. Whereas the variation of the diffracted high-harmonic intensities in Fig. 3c is qualitatively well reproduced in Fig. 3e, the calculated variation of the undiffracted signal in Fig. $3 \mathrm{~d}$ is found to significantly deviate from the experimental results in Fig. 3b. This discrepancy is removed when the increase in the number of high-harmonic emitters resulting from the dissociation of one molecule into two atoms is taken into account. ${ }^{[14]}$ In the future, accurate numerical calculations of the photorecombination matrix elements as a function of the dissociation coordinates would be desirable to further improve the theoretical modeling of these results.

A major benefit of the transient-grating measurements is that the simultaneous measurements of diffracted and undiffracted signals can further be used to extract both the amplitude and phase of harmonic emission from the excited molecules relative to the unexcited ones as shown previously. ${ }^{[13]}$ The high-harmonic amplitudes have been shown to provide additional insights into the evolution of the electronic structure as a function of the reaction coordinate, such as harmonic-order dependent minima that probe the internuclear separation of the molecule as it dissociates. ${ }^{[13,22]}$ The high-harmonic phases reveal the fast variation of the vertical ionization potential with internuclear separation and have also been found to be sensitive to the molecular orientation, as revealed by comparing experiments performed with either 


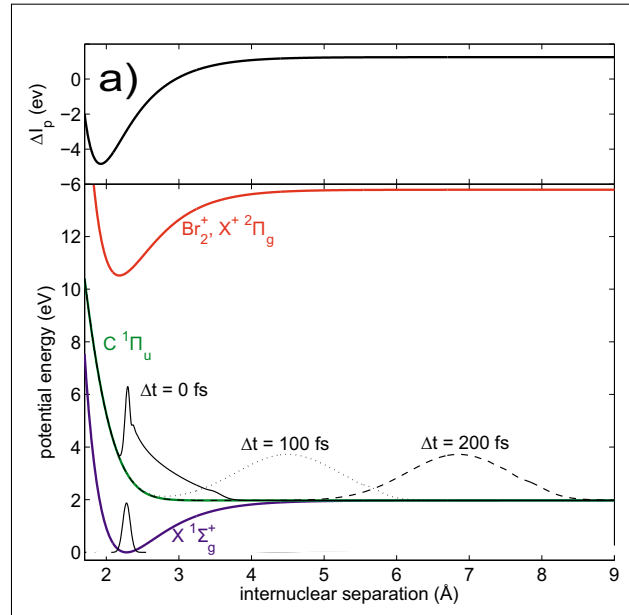

Fig. 3. a) Relevant potential-energy curves of $\mathrm{Br}_{2}$ and $\mathrm{Br}_{2}{ }^{+}$and $\Delta l_{\mathrm{p}}=I_{\mathrm{p}, \mathrm{C}}(\mathrm{R})-I_{\mathrm{p}, \mathrm{X}}(\mathrm{R})$. b) Experimentally observed undiffracted and c) diffracted signal from a TRHHS experiment on $\mathrm{Br}_{2}$ using 40 -fs excitation pulses centered at $400 \mathrm{~nm}$ and a $30-f$ s probe pulse centered at $800 \mathrm{~nm}$. The polarizations of the pump and probe pulses were parallel. Calculations from a simplified theoretical model show the undiffracted signals in panel d) and the diffracted signals in panel e).

parallel of perpendicularly polarized pump and probe pulses. ${ }^{[13]}$ Whereas the phase accumulated as a result of the difference in ionization potentials is independent of the molecular orientation in the limit of a single continuum, the phase contributed by the photorecombination transition moment can display a significant anisotropy that contains information on the electronic structure of the molecule.

As a second example we show how time-resolved high-harmonic spectroscopy can be generalized and extended to probe more complex photochemical dynamics. Nitrogen dioxide $\left(\mathrm{NO}_{2}\right)$ represents an interesting model system, because photoexcitation at $400 \mathrm{~nm}$ is known to be followed by an efficient ultrafast radiationless decay to the electronic ground state as the consequence of a conical intersection. Photoexcitation around $400 \mathrm{~nm}$ launches a nuclear wave packet in the $\tilde{A}^{2} \mathrm{~B}_{2}$ electronically excited state with the dominant configuration $(\ldots)\left(b_{2}\right)^{1}\left(a_{1}\right)^{2}$ (see Fig. 4a). The wave packet then moves toward the conical intersection that it can either cross, retaining its dominant electronic character or remain on the upper adiabatic surface thereby changing its electronic character to the dominant configuration (...) $\left(b_{2}\right)^{2}\left(a_{1}\right)^{1}$ of the $\tilde{X}^{2} \mathrm{~A}_{1}$ electronic ground state. Measurements of the photochemical dynamics of $\mathrm{NO}_{2}$ in a transient grating geometry are shown in Fig. 4b. The diffracted high-harmonic signals reveal pronounced oscillations with a period of about 100 fs. The undiffracted signals revealed much weaker oscillations with an opposite phase, ${ }^{[16]}$ barely visible on the scale of Fig. $4 \mathrm{~b}$. The diffracted signal reaches a maximum after $35 \mathrm{fs}$, subsequently decreases to a minimum around 70 fs and reveals another maximum around 130 fs. These ob-

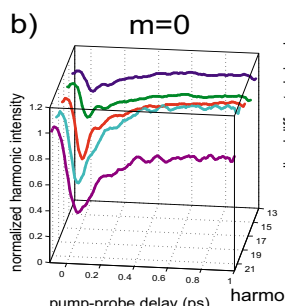

d)

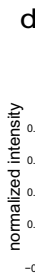

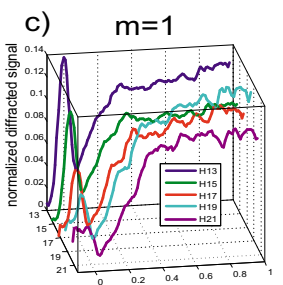

e)

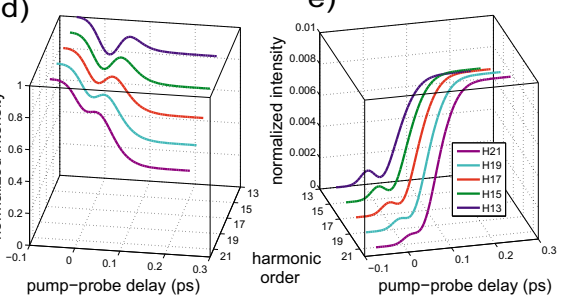

servations have been rationalized by wavepacket calculations in full dimensionality shown in Fig. 4c. Following photoexcitation, population is transferred to the $\tilde{A}^{2} \mathrm{~B}_{2}$ electronically excited state, followed by oscillations of the diabatic-state populations that are driven by a periodic motion along the $\mathrm{O}-\mathrm{N}-\mathrm{O}$ bending angle $\beta .[16,23,24]$ After $11 \mathrm{fs}$, the wave packet crosses the conical intersection for the first time mainly diabatically, i.e. it does not change its electronic character, whereas the second crossing is mainly adiabatic, resulting in a minimum of the diabatic excited-state population after 60 fs. These dynamics are reflected in the high-harmonic signals, most clearly in the diffracted signal as a consequence of the enhanced contribution of the excited-state emission to diffracted radiation that has been discussed in Section 2 . We have shown in refs. $[16,17]$ how the observables of TRHHS can be obtained from wave-packet calculations and have obtained a good agreement.

How do these findings compare to the results obtained in $\mathrm{Br}_{2}$ ? In the case of the photodissociation of $\mathrm{Br}_{2}$, the signal modulations are dominated by the variations of the vertical ionization potential because the width of the nuclear wave packet in coordinate space remains small during photodissociation as shown in Fig. 2. In the case of $\mathrm{NO}_{2}$ the spreading of the nuclear wave packet along both the bending and asymmetric-stretch coordinates is much more significant. ${ }^{[16,23]}$ Consequently the averaging over the nuclear wave packet covers large variations of the vertical ionization potential which suppresses the modulation resulting from the $\Delta I \tau$ phase efficiently. ${ }^{[16,17]}$ While the importance of the coordinate dependence of $I_{\mathrm{p}}$ as encountered in $\mathrm{Br}_{2}$ is likely to apply to photochemical dynamics occurring mainly along a single soft coordinate as we further demonstrate below, the situation encountered in $\mathrm{NO}_{2}$ is likely to be representative of multi-dimensional photochemical dynamics of polyatomic molecules. In such cases, the signal variation is dominated by the temporal variation of the electronic populations rather than by the variation of ionization potentials with internuclear coordinates.

As a last example, we discuss recent results on $\mathrm{CH}_{3} \mathrm{I}$, which is a prototypical

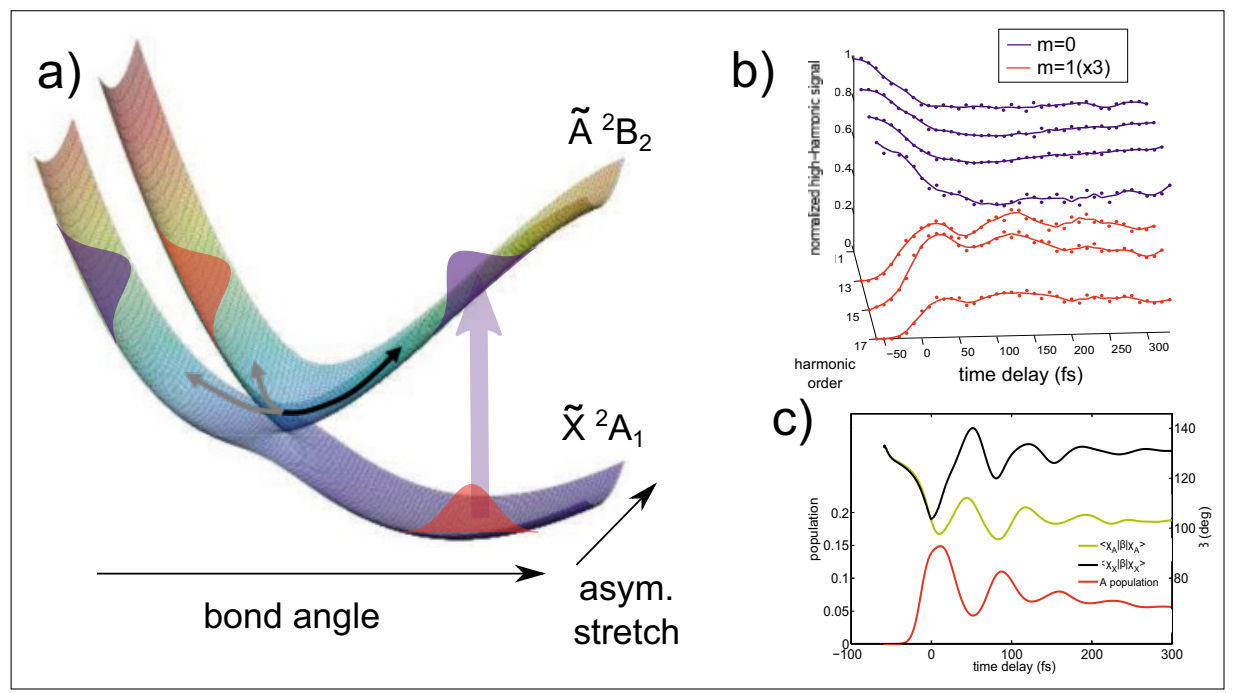

Fig. 4. a) Relevant potential-energy surfaces of $\mathrm{NO}_{2}$. b) Experimentally observed undiffracted (blue) and diffracted (red) signals from a TRHHS experiment on $\mathrm{NO}_{2}$ using 40 -fs excitation pulses centered at $400 \mathrm{~nm}$ and a $30-\mathrm{fs}$ probe pulse centered at $800 \mathrm{~nm}$. The polarizations of pump and probe pulses were perpendicular. c) Results of a three-dimensional wave packet calculation showing the population of the higher-lying diabatic state (A state) in red, and the expectation values of the $\mathrm{O}-\mathrm{N}-\mathrm{O}$ bond angle for the wave-packet components in the diabatic $\mathrm{X}$ state (black) and $A$ state (yellow). 
system for polyatomic photodissociation. We used pump pulses of 50-70 fs duration centered at $267 \mathrm{~nm}$, which transferred population from the $\tilde{X}^{1} \mathrm{~A}_{1}$ ground state to the dissociative ${ }^{3} \mathrm{Q}_{0+}$ state (Fig. 5). Photoexcitation prepares a dissociative nuclear wave packet that predominantly moves along the $\mathrm{C}-\mathrm{I}$ coordinate. The ${ }^{3} \mathrm{Q}_{0+}$ state correlates with $\mathrm{CH}_{3}$ in its electronic ground state and an iodine atom in the spin-orbit excited ${ }^{2} \mathrm{P}_{1 / 2}$ state. The photodissociation of $\mathrm{CH}_{3} \mathrm{I}$ has been studied in detail experimentally and theoretically (see e.g. refs. $[25,26]$ and references therein). The relevant potential surfaces are shown in Fig. 5a together with a one-dimensional wave packet propagation on the ${ }^{3} \mathrm{Q}_{0+}$ surface. The latter intersects the surface of the ${ }^{1} Q_{1}$ state as a function of the reaction coordinate. However, the population transfer to the ${ }^{1} Q_{1}$ state is small and has been neglected here. Fig. 5a further shows $\Delta I_{\mathrm{p}}$, the difference in vertical ionization potentials between the ${ }^{3} \mathrm{Q}_{0+}$ surface and the potential-energy minimum of the $\tilde{X}$ surface. The high-harmonic signal observed in the zeroth and first order diffraction is shown in Fig. 5b and c, respectively. The undiffracted signal is found to decrease followed by a recovery to an order-dependent level, as in the case of $\mathrm{Br}_{2}$. The diffracted signal however shows a temporal evolution that qualitatively differs from the results in $\mathrm{Br}_{2}$. The diffracted signals are found to increase during the excitation pulse, reaching a maximum after the peak of the pump pulse followed by a decrease to an asymptotic order-dependent level. These signals have been found to be well represented by the convolution of a single-exponential
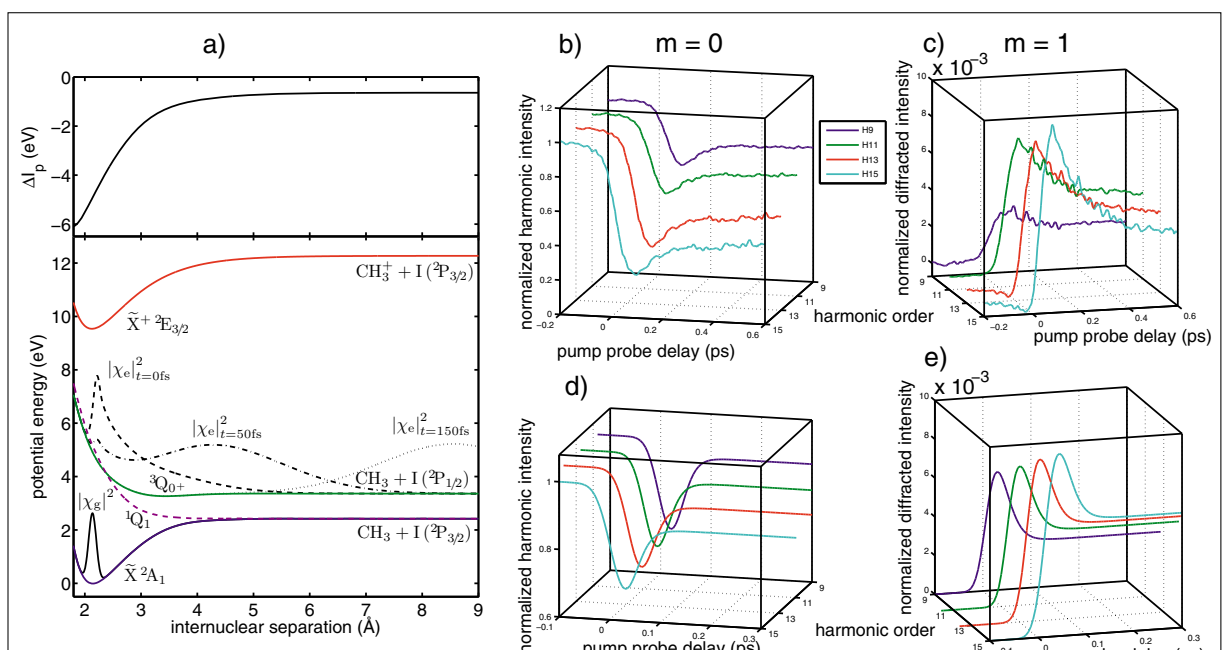

d)

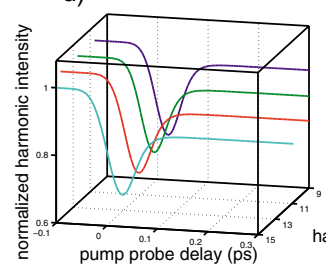

e)

decay with a Gaussian function. The exponential time constants were found to weakly depend on the harmonic order but to be consistent overall with previous measurements of the photodissociation time of $\mathrm{CH}_{3} \mathrm{I}^{[27]}$

We now turn to the theoretical modeling of these experimental results. We again use the same very simplified model as in the case of $\mathrm{Br}_{2}$. Only the variation of $\Delta I$ with the $\mathrm{C}-\mathrm{I}$ distance is taken into account. One-dimensional wave-packet propagation is performed on the ${ }^{3} Q_{0+}$ surface and the obtained nuclear wave packets are used to calculate the high-harmonic signals. While the spread of the nuclear wave packet mainly takes place along the soft $\mathrm{C}-\mathrm{I}$ coordinate, excitation of the $\mathrm{CH}_{3}$-umbrella and stretch modes during dissociation have also been observed. ${ }^{[25]}$ The signal observed in the zeroth and first order diffraction is nevertheless again qualitatively reproduced by our simplified model as shown in Fig. 5d and e. The initial drop of the undiffracted signal and the subsequent recovery to a signal level that decreases with increasing high-harmonic order is correctly reproduced. Similarly, the initial increase of the diffracted signal and its subsequent decay to a level that increases with growing harmonic order is also correctly predicted. From this result alone but even more conclusively by comparison with the results on $\mathrm{Br}_{2}$, we can thus validate the important contribution of the coordinate-dependent vertical ionization potential. However, the photodissociation of $\mathrm{CH}_{3} \mathrm{I}$ is not entirely adiabatic because of the presence of a conical intersection along the dissociation coordinate. As a consequence of the small

Fig. 5. a) Relevant potential-energy curves of $\mathrm{CH}_{3} \mathrm{I}$ and $\mathrm{CH}_{3} \mathrm{I}^{+}$and $\Delta I_{\mathrm{p}}=I_{\mathrm{p}, 300+}(\mathrm{R})-I_{\mathrm{p}, \mathrm{X}}(\mathrm{R})$. b) Experimentally observed undiffracted and c) diffracted signal from a TRHHS experiment on $\mathrm{CH}_{3} \mathrm{I}$ using 50 to $70 \mathrm{fs}$ excitation pulses centered at $267 \mathrm{~nm}$ and a $30-\mathrm{fs}$ probe pulse centered at 800 $\mathrm{nm}$. The polarizations of the pump and probe pulses were perpendicular. Calculations from a simplified theoretical model show the undiffracted signals in panel d) and the diffracted signals in panel e). non-adiabatic population transfer to the ${ }^{1} Q_{1}$ state ${ }^{[28]}$ and the relatively long excitation pulses, the present data do not reveal the surface crossing. Future measurements with shorter excitation pulses are expected to reveal the non-adiabatic dynamics.

\section{Conclusions and Outlook}

In this article, the main results and conclusions of the first experiments using timeresolved high-harmonic spectroscopy have been reviewed. The working principles and the main sensitivities of TRHHS can be summarized as follows. First, TRHHS is sensitive to the electronic structure of the reacting molecule through the amplitude and phase of the photorecombination transition moment. This is illustrated through minima in the amplitude of emission observed during the photodissociation of $\mathrm{Br}_{2}$ that are consistent with destructive quantum interference. ${ }^{[13,22]}$ Moreover, oscillations observed in the diffracted highharmonic yield in the case of $\mathrm{NO}_{2}$ are consistent with the evolution of the electronic character of the molecular wave packet as it crosses a conical intersection. ${ }^{[15-17,23]}$ Second, TRHHS is sensitive to the variation of the vertical ionization potential along the reaction coordinate. The relative phase of high-harmonic emission from excited and unexcited molecules has a contribution $\Delta I \tau$ (in atomic units) that is proportional to the difference of the vertical ionization potentials $\Delta I_{\mathrm{p}}$ and the electron excursion time $\tau$. This is illustrated by the results on both $\mathrm{Br}_{2}$ and $\mathrm{CH}_{3} \mathrm{I}$ where it was shown that a simplified theoretical model containing only the $\Delta I \tau$ contribution to the variation of the excited-state emission qualitatively reproduces the signal variations that were found to be very different for the two molecules. Third, TRHHS is also sensitive to temporal variations of the population of electronic states, even in cases where multi-dimensional wavepacket spreading prevents the observation of coordinate-dependent changes in the phase and amplitude of emission. This case was encountered in the photodissociation of $\mathrm{NO}_{2},{ }^{[15]}$ where the wave packet spreads considerably along both the bending and asymmetric-stretch coordinates. ${ }^{[16,17]}$ In this case, the signal modulations were found to reflect the temporal variation of the population of two states involved in the conical intersection, i.e. the evolution of the electronic character of the excitedstate wave packet as it crosses the conical intersection.

In the future, considerable additional insight will be obtained from improvements of both experimental and theoretical aspects. On the theoretical side, many improvements building on the present work 
can be made in the near future. Ionization rates can be calculated with one of the recent approaches, ${ }^{[29-31]}$ wave-packet calculations can be extended to the relevant dimensionality and photorecombination transition moments can be calculated using multi-electron treatments. ${ }^{[32]}$ The use of Dyson orbitals in modeling HHS ${ }^{[33,34]}$ gains its full importance when excited-state dynamics are described, since configurationinteraction effects are truly important in most electronically excited states.

On the experimental side, the information content can be extended by aligning or orienting the molecules (see e.g. ref. [35]) prior to photoexcitation. Performing the measurements reviewed in this article, in combination with independent measurements of the phase and amplitude of the unexcited molecules relative to a reference atom ${ }^{[36]}$ will provide all quantities needed to perform a reconstruction of the electronic structure as a function of time. However, the reconstruction method needs to be improved since previously used 'tomographic' approaches ${ }^{[5,12,37]}$ rely on some approximations of limited validity. ${ }^{[38,39]} \mathrm{A}$ self-consistent reconstruction approach such as the one studied in ref. [40] in a different context, appears to be a possible route to achieving a reliable reconstruction of electronic structure from experimental data.

Received: March 19, 2013
[1] P. B. Corkum, Phys. Rev. Lett. 1993, 71, 1994.

[2] M. Lewenstein, P. Balcou, M. Y. Ivanov, A. L'Huillier, P. B. Corkum, Phys. Rev. A 1994, 49, 2117.

[3] M. Lein, N. Hay, R. Velotta, J. P. Marangos, P. L. Knight, Phys. Rev. Lett. 2002, 88, 183903.

[4] T. Kanai, S. Minemoto, H. Sakai, Nature 2005, $435,470$.

[5] C. Vozzi, M. Negro, F. Calegari, G. Sansone, M. Nisoli, S. De Silvestri, S. Stagira, Nat. Phys. 2011, 7, 822 .

[6] J. Itatani, D. Zeidler, J. Levesque, M. Spanner, D. M. Villeneuve, P. B. Corkum, Phys. Rev. Lett. 2005, 94, 123902.

[7] N. L. Wagner, A. Wüest, I. P. Christov, T. Popmintchev, X. Zhou, M. M. Murnane, H. C. Kapteyn, Proc. Natl. Acad. Sci. USA 2006, 103, 13279.

[8] W. Li, X. Zhou, R. Lock, S. Patchkovskii, A. Stolow, H. C. Kapteyn, M. M. Murnane, Science 2008, 322, 1207.

[9] M. Lein, Phys. Rev. Lett. 2005, 94, 053004.

[10] S. Baker, J. S. Robinson, C. A. Haworth, H. Teng, R. A. Smith, C. C. Chirila, M. Lein, J. W. G. Tisch, J. P. Marangos, Science 2006, 312, 424.

[11] O. Smirnova, Y. Mairesse, S. Patchkovskii, N. Dudovich, D. Villeneuve, P. Corkum, M. Y. Ivanov, Nature 2009, 460, 972.

[12] S. Haessler, J. Caillat, W. Boutu, C. GiovanettiTeixeira, T. Ruchon, T. Auguste, Z. Diveki, P. Breger, A. Maquet, B. Carré, R. Taïeb, P. Salières, Nat. Phys. 2010, 6, 200.

[13] H. J. Wörner, J. B. Bertrand, D. V. Kartashov, P. B. Corkum, D. M. Villeneuve, Nature 2010, 466, 604 .

[14] H. J. Wörner, J. B. Bertrand, P. B. Corkum, D. M. Villeneuve, Phys. Rev. Lett. 2010, 105, 103002.

[15] H. J. Wörner, J. B. Bertrand, B. Fabre, J. Higuet, H. Ruf, A. Dubrouil, S. Patchkovskii, M. Spanner, Y. Mairesse, V. Blanchet, E. Mével, E. Constant, P. B. Corkum, D. M. Villeneuve, Science 2011, 334, 208.

[16] P. M. Kraus, Y. Arasaki, J. B. Bertrand, S. Patchkovskii, P. B. Corkum, D. M. Villeneuve, K. Takatsuka, H. J. Wörner, Phys. Rev. A 2012, 85, 043409.

[17] P. M. Kraus, H. J. Wörner, Chem. Phys. 2013, $414,32$.

[18] A. Tehlar, H. J. Wörner, Mol. Phys. 2013, accepted, doi:10.1080/00268976.2013.782439
[19] A. Rupenyan, J. B. Bertrand, D. M. Villeneuve, H. J. Wörner, Phys. Rev. Lett. 2012, 108, 033903.

[20] J. B. Bertrand, H. J. Wörner, H.-C. Bandulet, E. Bisson, M. Spanner, J.-C. Kieffer, D. M. Villeneuve, P. B. Corkum, Phys. Rev. Lett. 2011, 106, 023001.

[21] T. Kanai, E. J. Takahashi, Y. Nabekawa, K. Midorikawa, Phys. Rev. Lett. 2007, 98, 153904.

[22] H. J. Wörner, Chimia 2011, 65, 299.

[23] Y. Arasaki, K. Takatsuka, Chem. Phys. 2007, 338, 175.

[24] Y. Arasaki, K. Takatsuka, K. Wang, V. McKoy, J. Chem. Phys. 2010, 132, 124307.

[25] R. de Nalda, J. Durá, A. García-Vela, J. G. Izquierdo, J. González-Vázquez, L. Bañares, $J$. Chem. Phys. 2008, 128, 244309.

[26] C. R. Evenhuis, U. Manthe, J. Phys. Chem. A 2011, 115, 5992.

[27] D. Zhong, A. H. Zewail, J. Phys. Chem. A 1998, 102, 4031.

[28] S. P. Sander, J. P. D. Abbatt, J. R. Barker, J. B. Burkholder, R. R. Friedl, D. M. Golden, R. E. Euie, C. E. Kolb, M. J. Kurylo, G. K. Moortgat, V. L. Orkin, P. H. Wine, 'Chemical Kinetics and Photochemical Data for Use in Atmospheric Studies, Evaluation No. 17', Jet Propulsion Laboratory, Pasadena, 2011.

[29] M. Spanner, S. Patchkovskii, Phys. Rev. A 2009, $80,063411$.

[30] O. I. Tolstikhin, T. Morishita, L. B. Madsen, Phys. Rev. A 2011, 84, 53423.

[31] L. B. Madsen, O. I. Tolstikhin, T. Morishita, Phys. Rev. A 2012, 85, 53404.

[32] A. T. Le, T. Morishita, R. R. Lucchese, C. D. Lin, Phys. Rev. Lett. 2012, 109, 203004.

[33] R. Santra, A. Gordon, Phys. Rev. Lett. 2006, 96, 073606.

[34] S. Patchkovskii, Z. Zhao, T. Brabec, D. M. Villeneuve, Phys. Rev. Lett. 2006, 97, 123003.

[35] P. M. Kraus, A. Rupenyan, H. J. Wörner, Phys. Rev. Lett. 2012, 109, 233903.

[36] J. B. Bertrand, H. J. Wörner, P. Salières, D. M. Villeneuve, P. B. Corkum, Nat. Phys. 2013, 9, 174.

[37] J. Itatani, J. Levesque, D. Zeidler, H. Niikura, H. Pépin, J. C. Kieffer, P. B. Corkum, D. M. Villeneuve, Nature 2004, 432, 867.

[38] W. H. E. Schwarz, Angew. Chem. Int. Ed. 2006, 45, 1508.

[39] R. Santra, Chem. Phys. 2006, 329, 357.

[40] J. Xu, H.-L. Zhou, Z. Chen, C. D. Lin, Phys. Rev. A 2009, 79, 052508. 